\title{
Severe Uterine Hypertonus during Cesarean Section Relieved by Large Doses of Nitroglycerin
}

\author{
Roland Kaddoum, Cynthia Karam, Krystel Malek and Carine Foz* \\ Department of Anesthesia, American University of Beirut Medical Center, Beirut, Lebanon
}

Received: September 21, 2015; Accepted: October 01, 2015, Published: October 03, 2015

*Corresponding author: Carine Foz, Department of Anesthesia, American University of Beirut Medical Center, Beirut, Lebanon, Fax: 961-1745249; Phone: 961-1350000; E-mail: carinefoz@gmail.com

\begin{abstract}
A 36 years old female Gravida 3 Para 1 Aborta 1 presented at $35^{+2}$ weeks of gestation with increased frequency and intensity of her uterine contractions. An epidural catheter was inserted at L3-L4 interspace for labor analgesia. A decision was made for Cesarean delivery due to the transverse presentation of the baby. The epidural catheter was loaded by a bolus of $10 \mathrm{ml}$ lidocaine $2 \%, 5 \mathrm{ml}$ bupivacaine $0.1 \%$ and $1 \mathrm{ml}$ of fentanyl $50 \mathrm{mcg} / \mathrm{ml}$. Few minutes later; severe uterine hypertonus was noted impairing the delivery of the baby. Very large doses of nitroglycerin (a total dose of $1.3 \mathrm{mg}$ ) were needed for uterine relaxation.

Keywords: Uterine Hypertonus; Nitroglycerin; Epidural Analgesia
\end{abstract}

\section{Abbreviations}

CSE: Combined-Spinal Epidural; FHR: Fetal Heart Rate; NTG: Nitroglycerin

\section{Introduction}

Epidural analgesia is the most effective and innocuous technique for obstetrics [1] and has become the mainstay and the most popular method for providing pain relief during labor [2,3]. Low doses of long-acting local anesthetic in combination with low doses of fentanyl or sufentanil provide good quality analgesia and are safe for the mother and the fetus [1]. In our medical center, the standard epidural mixture consists of bupivacaine $0.1 \%$ and fentanyl $2 \mathrm{mcg} / \mathrm{ml}$ and is started once the diagnosis of active labor is established, at a rate titrated to analgesic effect.

We report a case of severe uterine hyperactivity following an epidural of fentanyl-bupivacaine in a patient undergoing Cesarean section, impairing the delivery of the baby and relieved by large doses of Nitroglycerin (NTG).

\section{Clinical Case}

A 36 years old woman Gravida 3 Para 1 Aborta 1, presented to our medical center at $34^{+3}$ weeks of gestation because of preterm contractions. Abdominal Ultrasound showed a vertex presentation of the baby. The patient was discharged home on Ritodrine $10 \mathrm{mg}$ orally every 4 hours along with IM injections (10mg), of which she received two doses.

The patient presented back at $35^{+2}$ weeks of gestation because of an increase in the intensity and frequency (every 3 $\mathrm{min}$ ) of her uterine contractions. The fetal heart rate was reactive with a baseline of 135 beats per minute and a good beat to beat variability.

After a decision was made to deliver the baby vaginally, an epidural catheter was inserted at L3-L4 using a 16G Tuohy needle and was advanced $4 \mathrm{~cm}$ in the epidural space. A test dose of 3 $\mathrm{ml}$ lidocaine $1.5 \%$ with $15 \mathrm{mcg}$ epinephrine was given and was negative as evidenced by no increase in maternal heart rate more than $20 \%$ above baseline and no motor blockade of the lower extremities. A bolus of $10 \mathrm{ml}$ of fentanyl $(5 \mathrm{mcg} / \mathrm{ml})$ was injected epidurally and an infusion of bupivacaine $0.1 \%$ with fentanyl $2 \mathrm{mcg} / \mathrm{ml}$ was started at a rate of $10 \mathrm{ml} / \mathrm{hr}$.

Oxytocin was not started at any time and a routine abdominal exam by Leopold's maneuver showed a transverse presentation of the baby that was confirmed by ultrasound. As a result, the decision was taken to deliver the baby via cesarean section 45 minutes after the epidural infusion was started. Therefore, a bolus of $20 \mathrm{ml}$ lidocaine $2 \%$ was injected in the epidural catheter to establish surgical anesthesia for Cesarean Section. A T4 sensory block was achieved as documented by the loss of pinprick sensation.

During cesarean section and while delivering the baby, the surgeon noted severe uterine hypertonus that trapped the leg of the baby and impaired his delivery, 10 minutes after the epidural bolus was given. He informed us about the problem and wanted immediate uterine relaxation. The patient's blood pressure was $154 / 90$ at the time, so she received $200 \mathrm{mcg}$ of Nitroglycerin (NTG) intravenously without noticeable effect. Then, two boluses of $300 \mathrm{mcg}$ each were also given but, the uterine rigidity persisted and the baby couldn't be delivered. Three minutes after the first NTG dose, a bolus of 500 mcg was given and a spectacular uterine relaxation was noted allowing the delivery of the baby, 1 minute after uterine incision. The patient's blood pressure was 
monitored every 1 minute and did not drop below 98/54 at any time. Apgar score at 1 and 5 minutes respectively was 2 and 7 and the baby's clinical condition ameliorated without any need for intensive care monitoring.

\section{Discussion}

Uterine hyperactivity is one of the undesirable side effects following labor analgesia, and may induce severe intrapartum fetal distress. It must be adequately detected and treated with intrauterine resuscitation techniques like maternal repositioning, intravenous fluid bolus, oxygen administration, and by the use of tocolytic agents [4]. The pain and stress of labor have been proven to result in large increase in circulating catecholamine, norepinephrine and epinephrine, which together decrease uterine contractility [5]. However, pain relief during effective regional analgesia modifies this pattern and causes an acute decrease in circulating maternal catecholamines, especially epinephrine, resulting in a transient imbalance in catecholamine levels that may lead to uterine hypertonicity and subsequent Fetal Heart Rate (FHR) abnormalities [6-8]. Other tentative explanations for the transient uterine hypercontractility are a direct stimulatory effect of the local anesthetic on the myometrial fibers and the local vasodilation resulting from sympathetic blockade that can lead to a transient higher amount of circulating oxytocic compound reaching the myometrium [9].

Parturients in more active labor have been shown to be at greater risk for uterine hyperactivity than those in early labor [10] which applies to our case since the patient presented with uterine contractions every 3 minutes. Many studies have shown that induction of effective labor analgesia might result in fetal bradycardia that is most likely related to an increase in uterine activity [11]. And while many studies have demonstrated that those changes in FHR and uterine tone are more common after intrathecal opioids [11-13], some others have shown that they occur with similar frequencies in association with any method of effective labor pain relief [14-16]. Norris et al found that in many cases, FHR abnormalities can be attributed to an increase in uterine tone, but these changes occur with similar frequencies irrespective of the method of labor analgesia used [10]. Moreover, Nielsen et al concluded that the incidence of clinically significant FHR abnormalities that can be due to an increase in baseline uterine tone is equivalent in patients receiving intrathecal sufentanil when compared to epidural bupivacaine within the first hour of administration [15]. The low Apgar score noted at 1 minute in our case can be then due to uteroplacental vascular insufficiency and thus FHR variable decelerations that have might resulted from the uterine hyperactivity that was noted.

Cohen et al. [6] showed that the estimated probability of occurrence of uterine hypertonus and fetal heart rate abnormalities was higher among patients with intense pain relief immediately after the block, strengthening the correlation between the speed of pain relief seen in Combined Spinal Epidural (CSE) and FHR abnormalities. Also, Abrao et al. [7] tested the hypothesis that CSE analgesia is associated with elevation of baseline uterine tone and FHR abnormalities when compared with the traditional epidural, and suggested that the occurrence of uterine hypertonus is even more frequent than observed in previous studies.

Friedlander reported the case of a 20-year-old parturient who experienced sustained uterine tetanus as detected by an intrauterine pressure catheter following subarachnoid fentanyl administration, necessitating delivery by Cesarean section after being only partially relieved by $0.25 \mathrm{mg}$ of terbutaline [17]. In our case, the patient experienced severe uterine hyperactivity following the epidural bolus of lidocaine. While most cases of uterine hyperactivity resolve spontaneously, resistant cases need immediate intervention. Those changes are usually reversible and respond to conservative management with short-term tocolytics [4]. Nitroglycerin (50-100 mcg intravenously) or terbutaline (0.1$0.25 \mathrm{mg}$ intravenously or subcutaneously) are most of the time effective in reversing the uterine changes $[10,17]$.

Intravenous nitroglycerin is commonly used for rapid and transient uterine relaxation in the setting of fetal distress or difficult delivery due to uterine hyperactivity during cesarean section [18]. Its ease of intravenous administration, rapid and short duration of action and predictability has been documented [19]. It can provide safe and effective uterine relaxation, with no adverse maternal or fetal effects when used in low doses [20]. NTG acts by the release of nitric oxide that relaxes uterine smooth muscles [21]. The intravenous dose of NTG required to attain the desired degree of uterine relaxation is extremely variable and there have been some controversies concerning the suitable dose $[18,20]$.

Small doses (60-180 micrograms) of intravenous NTG are associated with resolution of severe fetal distress related to uterine hyperactivity along with negligible side effects $[22,23]$.

Matsuda et al. [18] showed that the intravenous administration of 200 micrograms of NTG was effective for rapid uterine relaxation during cesarean delivery.

In our case, however very large doses of NTG $11.3 \mathrm{mg}$ total dose) were needed for the resolution of the uterine hyperactivity. The patient's uterine hypertonicity was not initially resolved by a bolus of 200 mcg of intravenous NTG which was found by many studies to be an effective dose of uterine relaxation. She required two additional intravenous boluses of $300 \mathrm{mcg}$ and it was not until a large bolus of $500 \mathrm{mcg}$ was given that effective uterine relaxation was obtained.

Although a total of $1.3 \mathrm{mg}$ of NTG was used, hypotension was not noted at any time.

\section{Conclusion}

Severe uterine hyperactivity following neuraxial blockade to relieve labor pain has been documented. Nitroglycerin has been shown to be a rapid and efficient tocolytic in reversing uterine hyperactivity. Although it is more common with intrathecal opioids, it has been documented after epidural opioids injections. 
Here we reported a case of severe uterine hypertonus following an epidural injection of local anesthetics and fentanyl that compromised the safety and impaired the delivery of the baby and that was not relieved by the usual doses of NTG. Although controversies around the suitable dose of NTG are present, such large doses were not used in any of the previous studies. To our knowledge, it's the first reported case of severe uterine hypertonus requiring very large doses of NTG for uterine relaxation.

\section{References}

1. Gomar C, Fernandez C. Epidural analgesia-anaesthesia in obstetrics. Eur J Anaesthesiol. 2000; 17(9): 542-58.

2. Harris AP, Michitsch RU. Anesthesia and analgesia for labor. Curr Opin Obstet Gynecol. 1992; 4(6): 813-7.

3. Kutlesic M, Kutlesic R. [Epidural analgesia in labor: specific characteristics, dilemmas and controversies]. Med Pregl. 2012; 65(910): 441-7.

4. Arnaout L, Ghiglione S, Figueiredo S, Mignon A. [Effects of maternal analgesia and anesthesia on the fetus and the newborn]. J Gynecol Obstet Biol Reprod (Paris). 2008; 37 Suppl 1: S46-55. doi: 10.1016/j. jgyn.2007.11.010.

5. Segal S, Csavoy AN, Datta S. The tocolytic effect of catecholamines in the gravid rat uterus. Anesth Analg. 1998; 87(4): 864-9.

6. Cohen SE, Cherry CM, Holbrook RH Jr, el-Sayed YY, Gibson RN, Jaffe RA. Intrathecal sufentanil for labor analgesia--sensory changes, side effects, and fetal heart rate changes. Anesth Analg. 1993; 77(6): 115560.

7. Abrao KC, Francisco RP, Miyadahira S, Cicarelli DD, Zugaib M Elevation of uterine basal tone and fetal heart rate abnormalities after labor analgesia: a randomized controlled trial. Obstet Gynecol. 2009; 113(1): 41-7. doi: 10.1097/AOG.0b013e31818f5eb6.

8. Segal S, Wang SY. The effect of maternal catecholamines on the caliber of gravid uterine microvessels. Anesth Analg. 2008; 106(3): 888-92, table of contents. doi: 10.1213/ane.0b013e3181617451.

9. Thiery M, Vroman S, de Hemptinne D, Yo Le Sian A, Vanderheyden K, Van Kets $\mathrm{H}$, et al. Elective induction of labor conducted under lumbar epidural block. II. Labor induction by amniotomy and intravenous prostaglandin. Eur J Obstet Gynecol Reprod Biol. 1977; 7(3): 181-200.

10. Norris MC, Fogel ST, Conway-Long C. Combined spinal-epidural versus epidural labor analgesia. Anesthesiology. 2001; 95(4): 913-20.

11. Clarke VT, Smiley RM, Finster M. Uterine hyperactivity after intrathecal injection of fentanyl for analgesia during labor: a cause of fetal bradycardia? Anesthesiology. 1994; 81(4): 1083

12.Van de Velde M, Teunkens A, Hanssens M, Vandermeersch E, Verhaeghe J. Intrathecal sufentanil and fetal heart rate abnormalities: a double-blind, double placebo-controlled trial comparing two forms of combined spinal epidural analgesia with epidural analgesia in labor. Anesth Analg. 2004; 98(4): 1153-9, table of contents.

13. Mardirosoff C, Dumont L, Boulvain M, Tramer MR. Fetal bradycardia due to intrathecal opioids for labour analgesia: a systematic review. BJOG. 2002; 109(3): 274-81.

14. Eberle RL, Norris MC, Eberle AM, Naulty JS, Arkoosh VA. The effect of maternal position on fetal heart rate during epidural or intrathecal labor analgesia. Am J Obstet Gynecol. 1998; 179(1): 150-5.

15. Nielsen PE, Erickson JR, Abouleish EI, Perriatt S, Sheppard C. Fetal heart rate changes after intrathecal sufentanil or epidural bupivacaine for labor analgesia: incidence and clinical significance. Anesth Analg. 1996; 83(4): 742-6.

16. Kuczkowski KM. Severe persistent fetal bradycardia following subarachnoid administration of fentanyl and bupivacaine for induction of a combined spinal-epidural analgesia for labor pain. J Clin Anesth. 2004; 16(1): 78-9.

17. Friedlander JD, Fox HE, Cain CF, Dominguez CL, Smiley RM. Fetal bradycardia and uterine hyperactivity following subarachnoid administration of fentanyl during labor. Reg Anesth. 1997; 22(4): 378-81.

18. Matsuda C, Hayashi S, Kinouchi K. [The effect of nitroglycerin 200 microg on uterine relaxation during cesarean delivery]. Masui. 2013; 62(4): 390-4.

19. Dufour P, Vinatier D, Puech F. The use of intravenous nitroglycerin for cervico-uterine relaxation: a review of the literature. Arch Gynecol Obstet. 1997; 261(1): 1-7.

20.Smith GN, Brien JF. Use of nitroglycerin for uterine relaxation. Obstet Gynecol Surv. 1998; 53(9): 559-65.

21.Segal S, Csavoy AN, Datta S. Placental tissue enhances uterine relaxation by nitroglycerin. Anesth Analg. 1998; 86(2): 304-9.

22. Mercier FJ, Dounas M, Bouaziz H, Lhuissier C, Benhamou D. Intravenous nitroglycerin to relieve intrapartum fetal distress related to uterine hyperactivity: a prospective observational study. Anesth Analg. 1997; 84(5): 1117-20.

23. Axemo $\mathrm{P}, \mathrm{Fu} \mathrm{X}$, Lindberg $\mathrm{B}$, Ulmsten $\mathrm{U}$, Wessen $\mathrm{A}$. Intravenous nitroglycerin for rapid uterine relaxation. Acta Obstet Gynecol Scand. 1998; 77(1): 50-3. 